\title{
Efectos de la Exposición de los Adolescentes a la Violencia en la Comunidad: El Proyecto MORE
}

\section{Effects of Youth's Exposure to Community Violence: The MORE Project}

\author{
Michele Cooley-Strickland, Tanya J. Quille, Robert S. Griffin, Elizabeth A. Stuart, Catherine P. \\ Bradshaw y Debra Furr-Holden \\ Johns Hopkins University, USA
}

\begin{abstract}
Resumen. La investigación sobre la exposición a la violencia crónica en la comunidad se centra en las minorías étnicas y en los colectivos más pobres y azotados por la delincuencia, mientras que los programas de tratamiento y prevención se centran en los perpetradores de la violencia, y no en los adolescentes, que son sus víctimas directas o indirectas. Las intervenciones de tratamiento y prevención en el ámbito escolar son necesarias en el caso de menores con un alto riesgo de exposición a la violencia en su comunidad. En este trabajo se describe el proyecto Multiple Opportunities to Reach Excellence (MORE), un estudio epidemiológico longitudinal centrado en las comunidades y que ha sido puesto en práctica en la actualidad para comprender mejor el impacto de la exposición a la violencia crónica en el vecindario sobre el funcionamiento emocional, conductual, de consumo de drogas y académico de los menores y adolescentes, con el objetivo último de identificar los factores maleables de riesgo y protección en los que se puede incidir mediante programas de prevención e intervención.

Palabras clave: consumo de drogas, externalización, internalización, niños y adolescentes, prevención, rendimiento académico.
\end{abstract}

\begin{abstract}
Research on chronic community violence exposure focuses on ethnic minority, impoverished, and crime-ridden communities while treatment and prevention focuses on the perpetrators of the violence, not on the youth who are its direct or indirect victims. School-based treatment and preventive interventions are needed for children at elevated risk for exposure to community violence. This paper describes The Multiple Opportunities to Reach Excellence (MORE) Project, a longitudinal, community epidemiological study currently being fielded to better understand the impact of children's chronic exposure to community violence on their emotional, behavioral, substance use, and academic functioning with an overarching goal to identify malleable risk and protective factors which can be targeted in preventive and intervention programs.
\end{abstract}

Keywords: academic, children and youth, community violence, externalizing, internalizing, prevention, substance use.

Para llegar a entender los complejos factores de riesgo y factores protectores asociados a los efectos de la exposición de los jóvenes a la violencia, se precisa de más investigaciones longitudinales basadas en muestras amplias y representativas de niños expuestos a distintos niveles de violencia en sus comunidades. Sin embargo, existen muy pocos estudios de este tipo. Este documento describe uno de dichos estudios que se está llevando a cabo en la actualidad, las teorías en las que se sustenta, sus objetivos y metodología, así como las implicaciones para futuros estudios e intervenciones de tratamiento y prevención. El objetivo es explicar los fundamentos de este ámbito de investigación y los retos a los que se enfrenta, y promover la intervención e investigación acerca de los efectos de la

Correspondencia: Michele Cooley-Strickland, Department of Mental Health, Johns Hopkins Bloomberg School of Public Health, 615 North Wolfe Street, Baltimore, MD 21205-2179, USA. E-mail: mсоoley@jhsph.edu violencia crónica en los vecindarios en los adolescentes. El nombre de este estudio epidemiológico longitudinal basado en la comunidad es The Multiple Opportunities to Reach Excellence (MORE) Project. Con este proyecto se pretenden corregir ciertas debilidades metodológicas de las investigaciones cross-seccionales y otros estudios menos rigurosos, y obtener una mayor comprensión del impacto que posee la exposición a la violencia en el vecindario sobre el funcionamiento emocional, conductual, académico y el consumo de drogas de los niños. Uno de los objetivos generales del estudio es identificar los factores de riesgo y los factores protectores sobre los que se puede incidir en posteriores programas de prevención e intervención.

La violencia en la comunidad es reconocida como uno de los mayores problemas de salud pública (Organización Mundial de la Salud, 2002). Sin embargo, la mayoría de las investigaciones sobre la violencia crónica en la comunidad se centran en aquellos que 
sufren un impacto más directo: las minorías étnicas, así como los grupos más pobres y más afectados por la delincuencia. La mayoría de la investigación e intervenciones de tratamiento y prevención existentes se centra en los perpetradores de la violencia y no en los jóvenes, que son sus víctimas directas o indirectas. El impacto que tiene en la salud pública vivir en comunidades violentas es importante, en especial en el caso de los niños y adolescentes. Entre los correlatos emocionales, conductuales y académicos se encuentran la ansiedad, depresión, conducta disrruptiva y agresiva, consumo de drogas, absentismo escolar y fracaso académico (e.g., Cooley-Quille, Boyd, Frantz y Walsch, 2001; Gorman-Smith y Tolan, 1998; Jenkins y Bell, 1994; Lorion, Brodsky y Cooley-Quille, 1999; Osofsky, Werers, Hann y Fick, 1993; Pynoos, Frederick, Nader y Arroyo, 1987; Schwab-Stone et al., 1999; Singer, Anglin, Song y Lunghofer, 1995), los cuales podrían tener un profundo impacto en el desarrollo del niño desde su infancia hasta la adolescencia e, incluso, a más largo plazo.

Son necesarias las intervenciones de tratamiento y prevención en el ámbito escolar para niños con alto riesgo de exposición a la violencia en su comunidad cuando esa exposición esté deteriorando su capacidad de desarrollarse adecuadamente y lograr buenos resultados académicos. Aunque se han producido importantes avances en la investigación sobre la violencia en la comunidad durante la pasada década, en general los estudios adolecen de muchos puntos débiles metodológicos (por ejemplo, el uso de instrumentos no contrastados psicométricamente, muestras seleccionadas por conveniencia, informes retrospectivos). De esta forma, la generalización de los resultados y la aplicabilidad de las conclusiones se minimiza (Schubiner, Scott y Tzelepis, 1993; Shakoor y Chalmers, 1991; Schwab-Stone et al., 1999). La investigación en este área ha sido en su mayor parte de carácter cross-sectional y existen muy pocos estudios que examinen los efectos longitudinales y en el desarrollo de la exposición sostenida a la violencia (DuRant, Cadenhead, Pendergrast, Slavens y Linder, 1994). Esto limita la capacidad de establecer la causalidad e identificar variables que podrían mediar en la asociación entre la exposición a la violencia en la comunidad y el ajuste (Cooley-Quille, Turner, y Beidel, 1995). Además, muchas de las investigaciones existentes se han centrado en muestras pequeñas o muy específicas (por ejemplo, delincuentes juveniles, varones de alto riesgo), han utilizado medidas de violencia en la comunidad no estandarizadas o no comparables y carecen de una base teórica sólida.

Gorman-Smith y Tolan (1998) recomiendan que se investigue el impacto de la exposición a la violencia en adolescentes que residen en distintos vecindarios y comunidades. El Proyecto MORE ha sido diseñado para determinar la prevalencia de la exposición total a la violencia en la comunidad entre niños que presentan distintos niveles de riesgo de exposición. Además, compara distintos estratos de vecindarios dentro de la misma ciudad con distintos índices (bajo, moderado, alto) de delitos violentos y de riesgo asociado de exposición a la violencia crónica en la comunidad. El estudio incluye varias vías de exposición a la violencia en la comunidad (es decir, a través de los medios de comunicación, denuncias, ser testigo o víctima de violencia, y la guerra/terrorismo), así como la perpetración de actos de violencia y el conflicto interparental. Entre los rasgos más significativos de este estudio destacamos: su diseño prospectivo longitudinal (tres paneles anuales), entrevistas con niños, padres/tutores y profesores, y un énfasis en los jóvenes en situación de riesgo según los distintos niveles de exposición a la violencia en la comunidad. Los métodos de medida permitirán investigar el modelo conceptual propuesto de los efectos emocionales, conductuales, de consumo de drogas y académicos en los adolescentes expuestos a violencia en su comunidad. Además, el contexto elegido para este proyecto (Baltimore city, Mariland, USA) es significativo, porque incluye una amplia variedad de índices de delincuencia en los vecindarios, hecho asociado con la exposición de los niños a la violencia en la comunidad (Selner-O’Hagan, Kindlon, Buka, Raudenbush y Earls, 1998).

\section{Violencia en la comunidad}

En torno al $80 \%$ de los niños que viven en áreas urbanas han sido testigos de violencia en su comunidad; hasta un $70 \%$ de éstos informan haber sido víctimas de esta violencia (Fitzpatrick y Boldizar, 1993; Gladstein, Rusonis y Heald, 1992; Kliewer, Lepore, Oskin y Johnson, 1998). La cultura juega un papel relevante en los niveles de violencia a la que los jóvenes están expuestos en su comunidad (Cooley, Turner, y Beidel, 1995). La violencia es particularmente severa en los vecindarios urbanos (e.g., Gladstein et al., 1992; Richters y Martinez, 1993). Se entiende por violencia en la comunidad cualquier acto deliberado con la intención de causar daño físico a una persona o personas de la comunidad (Cooley-Quille et al., 1995). Aunque las víctimas directas son obvias, las víctimas indirectas son mucho más numerosas. Se ven afectadas porque son: espectadores, testigos o familiares de las víctimas, o son conocedores de o experimentan ansiedad por la violencia potencial (Horn y Trickett, 1998; Lorion, 1998). La violencia crónica en la comunidad se ha extendido a múltiples ámbitos o grupos sociales y sus consecuencias afectan a un porcentaje importante de integrantes de la comunidad durante un prolongado periodo de tiempo (Lorion, 1998). Los jóvenes que viven en barrios pobres están expuestos a una mayor violencia que los que viven en barrios de un nivel socioeconómico medio-alto (Gladstein et al., 1992). 
Los jóvenes que viven en las áreas con mayores índices de delincuencia presentan los mayores niveles de exposición a la violencia; además se da la coincidencia de que estos barrios son también los más pobres de la ciudad (Selner-O'Hagan et al., 1998). A pesar de que unos altos índices de delincuencia aumentan las probabilidades de una exposición directa a la violencia en la comunidad, la exposición puede producirse por distintas vías (medios de comunicación, ser testigo de violencia, rumores, victimización, guerra/terrorismo) y se extiende más allá de los centros urbanos. La violencia en la comunidad afecta a todos los grupos raciales y étnicos (Cooley-Quille et al., 1995); sin embargo, los niños de minoría étnica, especialmente la afro-americana en los EEUU, se ven afectados de forma desproporcionada (Bureau of Justice Statistics, 1991; Christofel, 1990; Jenkins y Bell, 1994; SelnerO'Hagan et al., 1998).

No sólo resulta significativa la severidad de la exposición de los niños urbanos a la violencia en la comunidad, también lo es la cronicidad de ésta. Muchos estudios han revelado que los adolescentes afroamericanos están frecuentemente expuestos a una violencia en su comunidad crónica y severa (e.g., HintonNelson, Roberts y Snyder, 1996; Jenkins y Bell, 1994), más que a violencia episódica o hechos puntuales no recurrentes. Además, estos adolescentes en ocasiones no sólo están crónicamente expuestos a la violencia en la comunidad, sino también a otras formas de violencia (e.g., violencia intrafamiliar; Crouch et al., 2000; Hampton, Gelles y Harrop, 1989).

Los efectos acumulativos a largo plazo de una exposición crónica a la violencia en la comunidad y en otros entornos deberían ser observados durante los últimos años de niñez y al inicio de la adolescencia, para así poder determinar el impacto en el desarrollo del joven en diferentes ámbitos. La utilización de múltiples informantes y métodos para valorar la exposición de los jóvenes a la violencia en la comunidad mejora la fiabilidad de los trabajos con una única fuente de datos, que en la mayoría de los casos consiste en el auto-informe del niño. Los niveles de acuerdo entre padres e hijos en los cuestionarios sobre exposición a la violencia en la comunidad han sido bajos (Richters y Martinez, 1993). Los auto-informes de los jóvenes sobre la exposición a la violencia son sistemáticamente más altos que los de otros informantes (Ladd y Kochenderfer-Ladd, 2002; Schwartz y Gorman, 2003; Selner-O'Hagan et al., 1998). Así mismo, la exposición a la violencia en la comunidad que informan los niños es más alta que la que informan sus padres (e.g., Cooley y Boyd, 2009). Sin embargo, no está claro cuál de los dos resulta ser el informante más válido, teniendo en cuenta, por un lado, la premisa de que los jóvenes están mejor informados sobre sus propias experiencias con la violencia (Richters y Martinez, 1993; Schwarz, 1999) y por otro lado, que podría ser la percepción de la frecuencia y gravedad de la violencia lo que determina en mayor medida el impacto resultante de esa exposición.

\section{Exposición a la violencia en la comunidad y funcionamiento emocional, social y conductual.}

La teoría del estrés ha sido la principal base teórica empleada a la hora de investigar los efectos emocionales y conductuales de la exposición de los niños a la violencia en su comunidad (Horn y Trickett, 1998). Habitualmente, la violencia en la comunidad es el estresor identificado y se utiliza para predecir problemas de inadaptación. Se cree que la exposición crónica a la violencia en la comunidad posee un impacto negativo en varios aspectos de desarrollo y adaptación de los jóvenes (Attar y Guerra, 1994; Fitzpatrick y Boldizar, 1993; Jenkins y Bell, 1994; Martinez y Richters, 1993). Los jóvenes que crecen en entornos urbanos con altos índices de pobreza, superpoblación y violencia presentan un amplio rango de problemas de inadaptación, incluyendo la internalización de síntomas tales como ansiedad, síntomas de estrés postraumático, depresión, fracaso académico y absentismo escolar (e.g., Lorion et al., 1999; Osofsky et al., 1993; Singer et al., 1995). Los jóvenes con altos niveles de exposición a violencia en sus comunidades (por incidencia y/o gravedad) informan de forma significativa de un mayor nivel de angustia que aquéllos con una exposición menor (e.g., Fitzpatrick y Boldizar, 1993; Martinez y Richters, 1991).

En conjunto, los resultados revelan que la exposición de los niños a la violencia en la comunidad incrementa las probabilidades de que se desarrolle una internalización de síntomas (e.g., Fitzpatrick, 1993; Fitzpatrick y Boldizar, 1993; Martinez y Richters, 1993), aunque existen estudios que no han encontrado una relación positiva significativa (e.g., White, Bruce, Farrell y Kliewer, 1998). Las revisiones de la literatura generalmente revelan que cuando los datos proceden de una misma fuente, se da una relación lineal positiva entre los síntomas de ansiedad e internalización y la exposición de los niños a la violencia (Horn y Trickett, 1998), de forma que a mayor exposición, más problemático es el resultado (e.g., Pynoos et al., 1987).

Los jóvenes urbanos pobres están en riesgo de experimentar una serie de síntomas emocionales y conductuales simultáneamente, además de un pobre funcionamiento psicosocial; los problemas por conducta disruptiva (Gorman-Smith y Tolan, 1998) y la agresión son centrales (Tolan y Henry, 1996). La exposición a la violencia en la comunidad a la que están sometidos los niños varones afroamericanos e hispanos ha sido relacionada con unos mayores índices de conducta agresiva (Gorman-Smith y Tolan, 1998). Ser testigo o víctima de violencia en la comunidad se revela como un factor de riesgo de futuras agresiones (Attar y Guerra, 1994; Bandura, Ross y Ross, 1961; Cooley-Quille et 
al., 2001; DuRant et al., 1994). Además, para complicar aún más la direccionalidad de la relación, existen estudios que muestran que la violencia en la comunidad podría exacerbar la externalización de determinadas características conductuales (Gorman-Smith y Tolan, 1998).

Schwab-Stone y colaboradores (1999) analizaron la exposición a la violencia en un estudio longitudinal basado en la comunidad y descubrieron que la exposición a la violencia estaba asociada con la externalización conductual y la internalización de síntomas para ambos géneros y todos los grupos étnicos. La co-ocurrencia de síndromes psiquiátricos podría apuntar a una patogénesis compartida subyacente (Tolan y Henry, 1996). Horn y Trickett (1998) concluyeron que quizás no sea una contradicción que la exposición de los niños a la violencia esté asociada con problemas conductuales de externalización (agresión) e internalización (ansiedad y afectividad); ambos pueden darse simultáneamente. Aunque existen resultados contradictorios (e.g., Loeber y Keenan, 1994), la literatura sugiere que los jóvenes que manifiestan ansiedad comórbida y agresividad simultáneamente podrían estar en mayor riesgo de desajuste que los adolescentes que presentan o bien síntomas de ansiedad, o bien conductas agresivas (e.g., Ialongo, Edelsohn, Werthamer-Larsson, Crockett y Kellam, 1996; Kashani, Deuser y Reid, 1991; Ladd y Burgess, 1999). La ansiedad infantil puede ser un importante factor de riesgo de agresión ya que los niños ansiosos perciben mayor amenaza y hostilidad en las situaciones ambiguas que los niños no ansiosos (Kashani et al., 1991). Este resultado concuerda con la teoría del procesamiento de la información social (Crick y Dodge, 1994), que postula que una serie de sesgos en el procesamiento de la información social en situaciones ambiguas puede desencadenar un comportamiento agresivo.

Los investigadores están cada vez más interesados en los factores de cognición social que puedan mediar en la relación entre la exposición a la violencia en la comunidad durante la niñez y la conducta agresiva (Bradshaw y Garbarino, 2004; Guerra, Huesmann y Spindler, 2003). Varios estudios han revelado que los niños agresivos tienden a mostrarse hipersensibles ante las señales de amenaza, prestan atención de forma selectiva a estas señales y pasan por alto otros factores situacionales que hubieran podido influenciar la conducta de la persona (véase Crick y Dodge, 1994; Dodge y Pettit, 2003). Se cree que los niños agresivos poseen un sesgo de atribución hostil que influencia su interpretación de la situación, de forma que perciben una mayor hostilidad en el comportamiento ambiguo de otras personas. Podrían además manifestar un amplio repertorio de respuestas agresivas y creer que las respuestas agresivas son más efectivas para lograr el objetivo deseado que las respuestas prosociales. De acuerdo con la teoría de aprendizaje social (Bandura, 1973), ser testigo de violencia podría modelar la agre- sión como un método efectivo, normativo y justificado de resolver un conflicto o de obtener los fines deseados. Los jóvenes agresivos tienden a percibir su propia conducta agresiva como la respuesta adecuada contra las intenciones hostiles de los otros (Dodge y Somberg, 1987).

Otros investigadores sugieren que un deficiente control de las emociones también podría jugar un papel importante al aumentar el riesgo de que se produzcan mecanismos de afrontamiento inadaptados y conducta agresiva en niños que presencian episodios de violencia (Mushe-Eizenman et al., 2004). Un bajo control de las emociones podría contribuir a un mal procesamiento de las claves sociales y a que se produzca una conducta agresiva impulsiva en situaciones ambiguas y potencialmente conflictivas. Por lo tanto, es importante examinar la relación entre los sesgos negativos de procesamiento de la información social, el afrontamiento, y el control de las emociones como factores que posiblemente afectan la asociación entre la exposición a la violencia en la comunidad y la conducta agresiva. Una mayor comprensión de los factores que median en la asociación entre la exposición a violencia en la comunidad y el funcionamiento agresivo y el rendimiento académico podría ser parte esencial en el desarrollo de las intervenciones preventivas centradas en variables mediadoras socio-cognitivas relevantes.

\section{La exposición de los niños a la violencia en la comunidad y el funcionamiento académico y cognitivo}

No se han investigado lo suficiente los efectos cognitivos, educativos y de logro académico de la exposición de los niños a la violencia (Osofsky, 1995). Los estudios sobre el impacto de la violencia en el vecindario en el rendimiento escolar de los niños son necesarios y encomiables, sin embargo todavía no se ha desarrollado suficiente investigación empírica para evaluar directamente el rendimiento académico (Schwartz y Gorman, 2003). Entre los pocos estudios disponibles se encuentran aquéllos que evalúan el rendimiento académico percibido (e.g., Bowen y Bowen, 1999; Overstreet y Braun, 1999; Schwab-Stone et al., 1995; Schwartz y Gorman, 2003). Se cree que los distractores que experimentan los adolescentes al estar expuestos a violencia en su comunidad y escuela afectan su desarrollo cognitivo (Horn y Trickett, 1998). Las consecuencias a largo plazo aún están por explorar, aunque los índices de abandono escolar son de casi el 50\% para los grupos étnicos minoritarios históricamente en desventaja (Swanson, 2004), particularmente entre aquéllos residentes en áreas urbanas con los mayores índices de pobreza y violencia. Cada vez está más extendida la opinión, desde una perspectiva del ciclo vital, de que el abandono escolar no es un evento aislado, sino un proceso que se inicia en los primeros 
años de la niñez y que se halla influenciado por un cúmulo de factores (Alexander, Entwisle y Kabbani, 2001).

La exposición a la violencia en la comunidad ha sido asociada con déficit de atención, disminución del rendimiento cognitivo (Saltzman, 1996; Singer et al., 1995) y disminución del logro académico (Bell y Jenkins, 1991). Se ha sugerido que estas dificultades académicas se producen por un descenso de los niveles de concentración debido a pensamientos intrusivos sobre los sucesos violentos, los cuales podrían irse acumulando a lo largo del tiempo y mediante una exposición repetida (Bell, 1997; Horn y Trickett, 1998; Taylor, Tucker, Chatters y Jayakody 1997), así pues se hace necesario un modelo mediacional (Schwartz y Gorman, 2003). En un estudio cross-seccional con estudiantes de escuela elemental urbana, Schwartz y Gorman reforzaron la justificación de dicho modelo al comprobar que la violencia en la comunidad estaba asociada con un bajo rendimiento académico mediado por síntomas depresivos y conductas conflictivas. Los autores sugieren que la exposición a la violencia en las comunidades podría llegar a interferir en el desarrollo del niño de las capacidades de auto-regulación y control conductual (Schwartz y Gorman, 2003), lo cual concuerda con lo expuesto en la literatura sobre el procesamiento de información social (Dodge y Pettit, 2003). El género también puede poseer un efecto interactivo, dado que las chicas de barrios pobres traumatizadas por la violencia son expulsadas del colegio y arrestadas con mayor frecuencia (Lipschitz, Molenberghs, Fitzmaurice y Ibrahim, 2000).

Cuando los cuidadores de los jóvenes experimentan estresores importantes, como ocurre habitualmente con aquéllos que habitan en barrios con altos índices de delincuencia, disminuye su capacidad para fomentar el desarrollo cognitivo y social de sus hijos, lo cual es importante a la hora de gestionar los logros académicos de sus hijos. La investigación llevada a cabo por los autores de este trabajo ha revelado la existencia de una relación positiva entre la violencia en el vecindario y la expulsión escolar (es decir, suspensiones escolares, expulsiones; e.g., Boyd, Cooley, Lambert y Ialongo, 2003). Debido a que la tarea evolutiva más relevante para los niños es el éxito académico, es importante investigar directamente el impacto de la violencia en el vecindario sobre el rendimiento académico infantil.

\section{Violencia en la comunidad: Diferencias de edad y de desarrollo}

En las comunidades que experimentan con frecuencia delitos violentos, el número (porcentajes e incidentes) de jóvenes que se convierten en testigos de actos violentos aumenta a medida que se incrementa la edad media de los niños en la comunidad (Horn y Trickett,
1998). Así pues, cuanto más tiempo residen en esos barrios, con mayor frecuencia se encuentran los niños expuestos a la violencia, y de ahí proviene la caracterización de las comunidades "crónicamente" violentas. Son especialmente preocupantes los resultados de un estudio en el que se encontró niños expuestos a violencia crónica y severa en la comunidad a edades muy tempranas (Attar y Guerra, 1994; Gladstein et al., 1992). Por ejemplo, los niños de primer y segundo grado informaron estar expuestos a violencia en la comunidad a unos niveles similares a los de quinto y sexto grado; los niños pequeños también habían sido testigos de un alto nivel de violencia antes de entrar en la escuela elemental (Richters y Martinez, 1993). El ser testigos de violencia a cortas edades (de 3 a 5 años de edad) se reveló como un factor de riesgo de problemas de conducta (Shahinfar, Fox y Leavitt, 2000). Para empeorar aún más el problema, los padres o cuidadores siempre infravaloran la frecuencia de la exposición de sus hijos a la violencia y se considera que esto merma su capacidad de proteger a los niños (Cooley, Boyd y Grados, 2004; Richters y Martinez, 1993). Los resultados de las investigaciones apuntan a que la exposición de niños de corta edad, así como de sus tutores, a la violencia en su comunidad constituye un factor de riesgo significativo de desarrollo desajustado (Osofsky, 1995; Scheeringa y Zeanah, 1995). Por ejemplo, Linares et al. (2001) hallaron justificación para un modelo mediacional del impacto de la ansiedad materna (malestar general, síntomas de estrés postraumático) en la relación entre la exposición a la violencia en la comunidad y la internalización y externalización de problemas de conducta de los hijos. Aisenberg y Ell (2005) obtuvieron resultados similares y concluyeron que las investigaciones de violencia en la comunidad deberían ir más allá de un enfoque individualizado centrado en el niño y dirigirse hacia un enfoque basado en la comunidad y que integre al niño, los padres, la familia y la comunidad, para contextualizar de forma correcta los efectos de la exposición y determinar la consiguiente prevención e intervención de salud mental. Claramente, se justifica la investigación de modelos de intervención más comprehensivos (ansiedad parental/familiar que afecta la conducta de los hijos).

Los jóvenes de menor edad son más vulnerables a los resultados adversos asociados con la exposición a la violencia que los jóvenes más mayores (SchwabStone et al., 1999), pero podría darse una vulnerabilidad aún mayor en edades incluso más tempranas. Debido a que los adolescentes de entre 12 y 15 años son víctimas de delitos más frecuentemente que cualquier otra franja infantil de edad (Jenkins, 2001), es importante llevar a cabo estudios futuros sobre el impacto emocional, conductual y académico de la exposición a la violencia en los adolescentes antes de que se inicie ese periodo crítico de victimización intensificada. Con este fin, los años intermedios de la infan- 
cia son un importante momento del desarrollo para iniciar un estudio longitudinal. El periodo intermedio de la infancia es también el momento en que las pautas académicas negativas se fijan y estabilizan (Schwartz y Gorman, 2003).

\section{La violencia en la comunidad: diferencias de género}

Los varones por lo general informan de una mayor exposición a la violencia en la comunidad que las mujeres (Selner-O'Hagan et al., 1998). Por ejemplo, los chicos mayores informan haber presenciado sucesos violentos más severos y con mayor frecuencia que las chicas (Schubiner et al., 1993; Singer et al., 1995; Jenkins y Bell, 1994). Sin embargo, existen excepciones y en algunos estudios no se han encontrado diferencias de género (Attar, Guerra y Tolan, 1994; Farrell y Bruce, 1997; Uehara et al., 1996). También hay resultados contradictorios en cuanto a la existencia de diferencias de género en las reacciones emocionales y conductuales de los niños ante la exposición a la violencia. En dos estudios se concluyó que ambos sexos presentaban cifras similares de síntomas de estrés prostraumático tras la exposición a actos violentos (Pynoos et al., 1987; Schwarz y Kowalski, 1991). Los estudios han mostrado que las chicas informan de una mayor internalización de los síntomas (ansiedad, depresión, malestar emocional general) asociados con la exposición que los chicos (Farrell y Bruce, 1997; Fitzpatrick y Boldizar, 1994; Jenkins y Bell, 1994). Sin embargo, no se hallaron diferencias de género en los resultados emocionales de los niños mayores (Martinez y Richters, 1993).

La literatura cada vez más amplia de investigación sobre la violencia en las comunidades revela que las chicas podrían ser más vulnerables tanto a la internalización como a la externalización de problemas conductuales. Por ejemplo, un estudio con estudiantes de sexto grado reveló que ser testigo de violencia era un predictor de externalización de problemas de conducta en las chicas, pero no de internalización (Farrell y Bruce, 1997). Otro estudio con niños urbanos principalmente afroamericanos concluyó que en el caso de las chicas, la exposición a la violencia en la comunidad estaba significativamente relacionada con diversas formas de ansiedad, pero no en el caso de los chicos (White et al., 1998). Aunque existen claras diferencias en función de la edad en la exposición de los jóvenes a la violencia en la comunidad, el impacto del género está menos claro. Posiblemente, existirán efectos de interacción.

\section{Violencia en la comunidad y juventud: factores de protección}

Los estudios sobre los efectos de la exposición infantil a la violencia en la comunidad se han centrado principalmente en los factores de riesgo, y no en los de protección. En general, es la combinación de los factores de riesgo lo que predice qué niños desarrollarán un funcionamiento adverso, y es en esta combinación en la que se basa el modelo de índice de adversidad (Rutter y Quinton, 1977; Sameroff, Bartko, Baldwin, Baldwin y Seifer, 1998). Sin embargo, se sabe que algunos jóvenes expuestos a violencia en su comunidad poseen extraordinarias habilidades de afrontamiento (Fitzpatrick y Boldizar, 1993). A pesar de experimentar niveles similares de riesgo y vulnerabilidad, los distintos resultados (incluyendo la resiliencia) sugieren que los factores de protección atenúan los efectos del trauma (Rutter, 1987; Garmezy, 1993). Son factores que inciden directamente en la conducta, pero también moderan la relación entre el riesgo y resultados más saludables (Jessor, van den Boss, Vanderryn, Costa y Turbin, 1995). Tolan, Guerra y MontainiKlovdahl (1997) analizan los estilos particulares de afrontamiento que los niños de los barrios pobres deben desarrollar con el fin de "mantenerse fuera de peligro". Sin embargo, Tolan y colaboradores subrayan la importancia de distinguir entre el afrontamiento "de adaptación" y el afrontamiento "efectivo". En nuestra investigación, hemos encontrado que el primer tipo corresponde a estilos de afrontamiento dirigidos a cubrir las necesidades contextuales inmediatas del niño, pero podrían no ser prosociales y quizás pueden resultar incluso antisociales (por ejemplo, peleas físicas o robos como "solución" de un problema). El afrontamiento efectivo emplea medios prosociales que producen un cambio positivo, particularmente a largo plazo y en un ámbito social más amplio.

Otras conceptualizaciones más tradicionales de factores de protección en la literatura sobre el trauma y que inciden en la violencia en la comunidad incluyen la salud y adaptación emocional premórbida anterior a la exposición, padres altamente funcionales, y unas buenas relaciones familiares (Pynoos, Steinberg y Piacentini, 1999). El entorno familiar (por ejemplo, la comunicación, el afecto, la calidez) y el apoyo familiar son factores de protección en el caso de jóvenes expuestos a violencia familiar (Boney-McCoy y Finkelhor, 1995; Gorman-Smith y Tolan, 1998), pero también podrían ser considerados como factores generales de protección, como también lo son la inteligencia, la participación en la escuela y en actividades fuera del hogar, la religión, y la autoeficacia (Beardslee y Podorefsky, 1988; LaGreca, Silverman y Wasserstein, 1998; Luthar, Cicchetti y Becker, 2000; Resnick et al., 1997; Tiet et al., 1998). El impacto de un entorno escolar motivador (por ejemplo, con profesores cualificados y competentes, centros y aulas seguras) podría también actuar como elemento protector de los niños expuestos a violencia en la comunidad. Es interesante destacar que los jóvenes pueden experimentar numerosos factores de riesgo y de protección de forma simultánea (Jessor et al., 1995). Quizás la 
combinación de uno y otro tipo de factores podría explicar por qué algunos jóvenes evolucionan de forma relativamente saludable, mientras que otros evidencian unas significativas dificultades emocionales, conductuales y/o académicas.

A pesar de que se conocen las consecuencias de inadaptación asociadas a la exposición a violencia en la comunidad, existe poca investigación que examine factores combinados de riesgo y de protección asociados con la exposición infantil a la violencia en la comunidad (Boyd et al., 2003). Un estudio en el que se investigaba si las características individuales protegían a los niños de la exposición a la violencia en la comunidad reveló que en el caso de los chicos agresivos, los bajos niveles de ansiedad les protegían de exposiciones posteriores (Boyd et al., 2003). Se precisan más estudios para llegar a comprender totalmente los mecanismos de protección y de riesgo relacionados con la exposición a violencia en la comunidad. Por ejemplo, las actividades prosociales podrían poseer una naturaleza protectora porque provocan la disminución de las ocasiones de interactuar con grupos de jóvenes desviados, proporcionan apoyo social y aumentan la autoeficacia. En relación con esto, el bienestar de los niños sufre en mayor medida en el caso de aquéllos con bajos niveles de apoyo social o altos niveles de tensión social (definidos por ser aquéllos que carecen de redes sociales que les permitan hablar acerca de la violencia; Kliewer et al., 1998). Los jóvenes necesitan más apoyo que los adultos porque poseen menos habilidades para comunicar sus preocupaciones con relación a su trauma y poseen menos recursos de apoyo formales e informales y de afrontamiento psicológico (Kliewer et al., 1998).

Se podría dar un factor de protección adicional en la fuerza interna que se manifiesta a través de las creencias y prácticas religiosas. Algunos estudios se han centrado en las conexiones entre la participación en actividades religiosas y/o espirituales y la salud física y mental (Dossey, 1993; Koenig, 1997). Los estudios generalmente revelan que la religión y la espiritualidad poseen una modesta relación con el bienestar emocional (Ellison, 1991). Se han propuesto distintos mecanismos para explicar esta relación, tal como el compromiso religioso/espiritual, el cual proporciona: un significado u objetivo vital; paz interior; conexión con otros y la comunidad (Walters y Bennett, 2000). Explorar los factores de riesgo y de protección asociados es básica para superar la fase de investigación epidemiológica y avanzar hacia el tratamiento y la prevención.

\section{Violencia en la comunidad, juventud y ansiedad}

El trastorno de estrés postraumático (TEPT) es el trastorno de ansiedad con síntomas fisiológicos más ampliamente conocido, aunque todos los tipos de trastorno de ansiedad afectan los sistemas de respuesta fisiológica, conductual y cognitiva (Kendall y Ham- men, 1995). El análisis de cada una de estas áreas es importante para entender el origen del TEPT (Jones y Barlow, 1990) y otros síntomas de ansiedad e internalización. Los síntomas de estrés postraumático podrían servir para ilustrar la conexión entre la violencia en la comunidad y la ansiedad, porque el inicio del TEPT se halla necesariamente precedido por un estresor externo. Algunos estudios previos apoyan la relación entre los síntomas del TEPT y la exposición de los jóvenes a la violencia en la comunidad (Pynoos et al., 1987; Schwarz y Kowalski, 1991). La exposición a un trauma externo provoca una actividad exagerada de los neurotransmisores y se halla relacionada con la agresividad, la hipersensibilidad (Turner, Beidel y Cooley-Quille, 1997), y la hiperexcitación fisiológica y subjetiva (Keane, Gerardi, Lyons y Wolfe, 1988). Los factores biológicos están involucrados pero no pueden explicar por sí solos por qué las personas desarrollan TEPT tras haber estado expuestas a traumas, mientras que otras no (Turner et al., 1997). Estudios tempranos revelaron que los adolescentes que residen en barrios pobres urbanos presentan presiones sanguíneas más altas que los adolescentes que residen en áreas periféricas o rurales (Thomas y Groer, 1986), independientemente de la raza (Burns, Morrison, Khoury y Glueck, 1980). La vida en áreas urbanas es descrita como menos agradable estéticamente, más ruidosa y más hacinada (Thomas y Groer, 1986). Es interesante señalar un estudio que llevamos a cabo en el que encontramos que los adolescentes urbanos de mayor edad con altos niveles de exposición a violencia en la comunidad presentaban presiones sanguíneas en reposo más bajas que los adolescentes con una menor exposición (Cooley-Quille y Lorion, 1999).

Es posible que los adolescentes mayores, crónica y repetidamente expuestos a sucesos violentos, se habitúen o desensibilicen (Fitzpatrick y Boldizar, 1993). Por ejemplo, las señales de peligro (e.g., sirenas de policía o ambulancia, detonaciones de arma) podrían ocurrir con tanta frecuencia que el joven finalmente aprenda a no reaccionar con una respuesta de "ataque o huída", acostumbrándose al miedo. Por el contrario, los jóvenes de menor edad o con una baja frecuencia de exposición evaden/evitan los estímulos que producen ansiedad, una conducta que ayuda a incrementar o mantener la intensidad de la respuesta de miedo (Turner et al., 1997). El modelo de habituación o extinción del miedo se encuentra apoyado por un considerable cuerpo de literatura que revela que el contacto prolongado a los estímulos que producen el miedo provoca un aumento de la reactividad fisiológica y la angustia subjetiva. Con una exposición repetida, la reactividad fisiológica y la ansiedad son seguidas por descensos de la excitación y el miedo (es decir, la respuesta se habitúa o se extingue; Mowrer, 1960). El problema reside en si los niños con altos niveles de violencia en su comunidad aprenden a desensibilizarse a los indicios de peligro, pero no aprenden habilidades de afrontamiento prosocial para gestionar su ansiedad. 
Algunos miedos son evolutivamente inapropiados en casi todas las circunstancias (Leonard, Goldberger, Rapoport, Cheslow y Swedo, 1990), mientras que otros son de adaptación o de protección (Marks, 1987). Los profesionales clínicos e investigadores deben diferenciar entre la ansiedad patológica y el miedo evolutivo normal (March y Parker, 1999). Los miedos y la ansiedad asociados a la vida en barrios pobres urbanos caracterizados por la pobreza, la violencia y la escasez de recursos plantean un reto a la hora de determinar si los miedos son razonables o de adaptación. Algunos comentarios realizados por niños que residen en los barrios pobres revelan miedos agudos a recibir algún tipo de daño (por ejemplo, ser raptados, ser heridos por bala); sus miedos son incluso mayores en cuanto al daño que pueda recaer sobre miembros de su familia (Cooley, Boyd y Grados, 2004). Algunos cumplen los criterios de umbral de Trastorno de Ansiedad por Separación (TAS), a pesar de tener más edad que los casos típicos de TAS infantil. Una preocupación constante por la seguridad o salud de las personas que quieren afecta probablemente a la habilidad de los niños de barrios pobres a la hora de desarrollar un funcionamiento evolutivamente apropiado, académicamente exitoso y saludable (Cooley et al., 2004).

\section{La violencia en la comunidad y los jóvenes: sintomas depresivos}

La depresión es un problema significativo a través de grupos raciales, étnicos y socioeconómicos, pero prevalece en aquéllos con un estatus socioeconómico bajo (Beardslee et al., 2003). Las minorías étnicas están sobre-representadas en los grupos con un bajo estatus socioeconómico (Bruce, Takeuchi y Leaf, 1991). Los niños urbanos pobres son los más vulnerables a desarrollar la internalización y externalización de problemas de conducta (McKay, Stoewe, McCadam y Gonzales, 1998; Tolan y Henry, 1996). Los alumnos de escuela elemental podrían experimentar síntomas y trastornos depresivos similares a los de los adultos (Weisz, Weiss, Wasserman y Rintoul, 1987). Los investigadores han encontrado asociaciones moderadamente fuertes entre la exposición de los niños a violencia en la comunidad y los síntomas de depresión, incluyendo pensamientos intrusivos, baja energía, y motivación limitada (Osofsky, 1995; Schwartz y Gorman, 2003).

La depresión es un trastorno que debe ser entendido desde distintas perspectivas: es un trastorno biológico, pero que afecta profundamente el funcionamiento y las relaciones familiares; los factores etiológicos son tanto genéticos como ambientales (Beardslee et al., 2003). Los hijos de padres con trastornos de estado de ánimo tienen cuatro veces más probabilidades de desarrollar trastornos de estado de ánimo que los niños en familias sin dolencias parentales; se han encontrado índices de depresión de hasta el 50\% en adolescen- tes y jóvenes adultos con padres gravemente enfermos (Beardslee, Versage y Gladstone, 1998). Otros factores potenciales significativos en el desarrollo y persistencia de la depresión incluyen: eventos vitales adversos; racismo y prejuicios, aunque sean experimentados de forma encubierta por las minorías étnicas/raciales; y la pobreza (e.g., Beardslee et al., 2003; Brown, Ahmed, Gary y Milburn, 1995; Turner y Lloyd, 1999). Los niños de minoría étnica que residen en entornos urbanos pobres están en riesgo de experimentar niveles crónicos de violencia en el vecindario (Barreto y McManus, 1997) y sus padres/tutores podrían limitar sus actividades fuera del hogar en un intento de protegerles de cualquier daño (McAlister-Groves, Zuckerman, Marans y Cohen, 1993). Sin embargo, el aislamiento social está asociado con la depresión y otros síntomas de internalización, de manera que estas restricciones podrían truncar el desarrollo emocional de los niños (Beardslee et al., 2003) y la salud física, e incrementar su vulnerabilidad a la depresión y otros problemas afectivos.

Los síntomas de depresión también podrían darse debido a la adversidad ambiental, como lo es ser víctima de violencia, el duelo por la pérdida de alguien, o tener a múltiples familiares de primer grado con el trastorno (Beardslee et al., 2003). Otros factores de riesgo son una baja autoestima, la indefensión, pertenecer al sexo femenino, y la pobreza (Institute of Medicine, 1994). La exposición de los jóvenes a la violencia en la comunidad ha sido relacionada con síntomas del trastorno (e.g., Kliewer et al., 1998; Martinez y Richters, 1993) pero la forma de manifestarse este trastorno podría estar relacionada con el tipo de violencia que experimentan los jóvenes (Cooley-Quille et al., 1995; McAlister-Groves et al., 1993). Por ejemplo, la exposición de los niños a formas puntuales (es decir, no recurrentes) de violencia en la comunidad podría estar relacionada con trastornos internos (por ejemplo, síntomas de ansiedad, depresión, somatización y aislamiento), mientras que la exposición a la violencia crónica en la comunidad podría estar relacionada con la externalización de conductas (por ejemplo, agresión, comportamiento problemático; Cooley-Quille et al., 1995). Este resultado diferencial podría ser una manifestación del aprendizaje vicario, de forma que presenciar altos niveles de violencia en la comunidad modela las conductas agresivas y de externalización (Cooley-Quille et al., 1995). Sin embargo, otras explicaciones alternativas incluyen los principios conductuales de refuerzo y castigo. En concreto, la internalización de síntomas (por ejemplo, la depresión, el aislamiento, o las dolencias somáticas) podrían ser rechazados (es decir, ser susceptibles al castigo) por los habitantes de comunidades con un alto índice de violencia, al ser estos síntomas percibidos como debilidades, haciendo así a los jóvenes vulnerables y blancos fáciles para futuras victimizaciones (Earls, 1991; Barretto y McManus, 1997). 


\section{Violencia en la comunidad y juventud: consumo de drogas}

Los índices de prevalencia de la iniciación en el consumo de drogas por raza/etnia indican que, mientras los afroamericanos se inician con menor frecuencia que los caucásicos en el consumo de tabaco y alcohol antes de los 13 años, están en mayor riesgo de iniciarse en el consumo de la cocaína y marihuana a edades más tempranas (Everett, Giovino, Warren, Crossett y Kann, 1998). Los datos de prevalencia de un inicio temprano en el consumo de drogas son escasos. Sin embargo, hay estadísticas descriptivas con algunas limitaciones sobre el consumo de drogas y su progresión. Por ejemplo, Wills, Sandy y Yaeger (2001) estudiaron niños de escuela elemental de una comunidad mixta urbana-suburbial (edad media 11.8 años; $27 \%$ de afroamericanos). Alrededor de un cuarto (24\%) de los niños informó haber fumado uno o dos cigarros y un $1 \%$ informó fumar al menos una vez al mes. En cuanto al consumo de alcohol, casi un tercio (30\%) de los alumnos de escuela elemental informaron haber bebido alcohol una o dos veces, y un $2 \%$ informó beber al menos una vez al mes. En cuanto a la marihuana, un $2 \%$ informaron haberla probado una o dos veces, y menos de un $1 \%$ informaron fumarla de forma regular (Wills et al., 2001). En otro estudio se examinó la prevalencia del consumo de alcohol y drogas entre los niños de tres ciudades: Denver, Pittsburgh y Rochester (Huizinga, Loeber y Thornberry, 1993). Los autores hallaron una alta frecuencia de iniciación a las drogas antes de la adolescencia. Los índices de Denver incluían las áreas con mayor riesgo; para el grupo de niños de siete años de edad: el $15.3 \%$ de los niños y el $9.7 \%$ de las niñas informaron haber bebido alcohol al menos una vez, mientras que el $1.2 \%$ de los niños y un $0.7 \%$ de las niñas de siete años informaron haber fumado marihuana una o más veces (Huizinga et al., 1993).

En un estudio preliminar realizado por el primer autor antes citado en el que se evaluaron los estilos de afrontamiento de los niños de barrios pobres, los alumnos de quinto grado afroamericanos informaron consumir drogas cuando "se enfrentaban a dificultades o se sentían tensos". Casi un cuarto (22.9\%) de los niños de 11 y 12 años informaron haber fumado tabaco y un $17.1 \%$ informó beber alcohol en ocasiones para ayudarles a "sobrellevar el estrés". Las investigaciones sobre los resultados asociados a la exposición a la violencia en la comunidad deberían abordar el consumo de drogas. Los niños podrían no considerarse a sí mismos "consumidores de drogas" fuera de contexto (e.g., no asociado con el grupo de iguales o con el estrés). Una ventaja de realizar los estudios a partir de los años intermedios de la infancia es que permite la identificación de variables de intervención y moderación que puedan influir en el inicio y persistencia del consumo de drogas.

Se ha relacionado el trauma con el abuso en el consumo de drogas por parte de los adolescentes (Kilpa- trick et al., 2000). Varios estudios revelan que el estrés postraumático es un factor de riesgo de desarrollo y cronicidad de la depresión y el consumo de drogas (Bolton et al., 2000; Giaconia y Reinherz, 1995; Kilpatrick et al., 2000). Se asocian problemas de conducta (e.g., agresión, conductas peligrosas) con el inicio del consumo de drogas (Epstein, Botvin, Diaz, Williams y Griffin, 2000). Un compendio exhaustivo de las posibles causas de la relación entre el consumo de drogas y la violencia fue presentado por Mulvey y colaboradores (Mulvey et al., 2006): 1) El consumo de drogas produce violencia ya sea directamente (por ejemplo, por medio de la desinhibición) o indirectamente (e.g., violencia condicionada por asociación con iguales agresivos; insuficientes habilidades de afrontamiento; estresores vitales); 2) Las experiencias con la violencia aumentan la probabilidad de consumo de drogas (e.g., consumo de drogas como respuesta de afrontamiento); o 3) La relación es falsa (es decir, un factor terciario aumenta la manifestación de consumo de drogas y/o agresividad) (Parker y Auerhahn, 1998).

Los patrones de desarrollo del consumo de drogas podrían variar según la edad, el género, la etnia, la clase social y las condiciones ecológicas, culturales e históricas (Kandel, Kessler y Marguiles, 1978). Ciertos factores de riesgo de iniciación a las drogas ejercen efectos diferenciales según el género y la etnia (Brunswick y Messeri, 1984; Ellickson y Morton, 1999). En cuanto a los adolescentes urbanos afroamericanos, se ha demostrado que variables de múltiples ámbitos (e.g., historia personal, logro escolar, orientación de la familia o los iguales, tendencias psicogénicas, actitudes y conductas de salud) influencian el inicio en el consumo de tabaco (Brunswick y Messeri, 1984). Sin embargo, los únicos predictores del "alto" consumo de drogas de los jóvenes afroamericanos fueron las influencias sociales que promueven el consumo de drogas y las intenciones de consumirlas (Ellickson y Morton, 1999).

La direccionalidad de la asociación entre consumo de drogas y un bajo rendimiento escolar no está del todo clara (Bryant, Schulenberg, Bachman, O’Malley y Johnston, 2000). Los investigadores encontraron que entre los alumnos de $8^{\circ}$ y $12^{\circ}$ grado, las experiencias adversas escolares (es decir, mal comportamiento escolar, pobre logro académico) precipitaban el consumo de tabaco (Bryant et al., 2000). Otro estudio reveló resultados similares entre los afroamericanos; aquéllos que abandonaban la escuela tenían mayores probabilidades de inyectarse drogas al llegar a la edad adulta (Obot y Anthony, 2000). Sin embargo, en otro estudio no se encontró que las notas bajas predijeran el consumo de sustancias en el grupo de alumnos de instituto afroamericanos, pero sí en el caso de los alumnos asiáticos (Mackesy-Amiti y Fendrich, 2000). La mayoría de investigadores recomiendan que se evalúen las relaciones entre las conductas problemáticas de los adolescentes (e.g., fracaso académico, con- 
sumo de alcohol y marihuana) a medida que cambian con el paso del tiempo, identificando particularmente los factores de riesgo y protección que inciden en las conductas no deseadas (Duncan, Duncan y Strycker, 2000).

\section{El Proyecto MORE}

En el Proyecto MORE se intenta dar respuesta a cinco preguntas principales: 1) ¿Cuál es la prevalencia de la exposición de los niños urbanos a la violencia en la comunidad? 2) ¿Cuáles son los factores de riesgo asociados a la exposición de los niños a violencia en la comunidad? 3) ¿Cuáles son los factores de protección asociados a la prevención de la exposición de los niños a la violencia en su comunidad? 4) ¿Cuáles son los resultados negativos de la exposición de los niños a la violencia en la comunidad? 5) ¿Qué factores protegen de los resultados negativos en los niños expuestos a violencia en su comunidad? Cada una de estas preguntas principales será, además, analizada por estrato de violencia en el vecindario (estratos bajos vs. moderados vs. altos o bajos-moderados vs. altos), por género, por edad y, cuando se considere apropiado, por informante (niño, padre, profesor).

El modelo conceptual que proporciona las bases para el Proyecto MORE se ilustra esquemáticamente en la Figura 1.
La muestra del Proyecto MORE se compone de 746 alumnos, sus padres/tutores y profesores (ver CooleyStrickland, Quille, Griffin, Stuart, Bradshaw y FurrHolden, 2009, para una explicación exhaustiva del reclutamiento de la muestra y los aspectos metodológicos de la investigación). El reclutamiento duró un año y medio académico y se realizó entre alumnos de ocho a doce años de edad que asistían a seis escuelas elementales públicas urbanas situadas en tres comunidades de Baltimore, Maryland, con índices bajos, moderados y altos de delincuencia en el vecindario. Para evitar sesgos de selección entre los sujetos, la raza/etnia no se utilizó como criterio de selección. Los criterios de selección de alumnos durante el reclutamiento de la muestra fueron: 1) Alumnos inscritos en jornada escolar completa en una de las seis escuelas elementales públicas identificadas de la Ciudad de Baltimore durante el otoño de 2006 ó 2007; 2) con edades entre los 8 y 12 años inclusive; 3 ) hablar inglés y vivir con un padre/tutor angloparlante. Los criterios de exclusión fueron: 1) Presencia de enfermedades médicas o neurológicas graves (e.g., epilepsia, traumatismo craneal interno) o retraso mental que impidiesen la realización de la entrevista; o 2) no residir con al menos un padre o tutor legal. Los instrumentos de evaluación del Proyecto MORE fueron seleccionados por ser apropiados para las edades de los sujetos, por sus propiedades psicométricas, por la disponibilidad de normas, y a ser posible, la pertinencia de que sean usados

Figura 1. Modelo Conceptual de los efectos de la exposición de los niños a la violencia en la comunidad

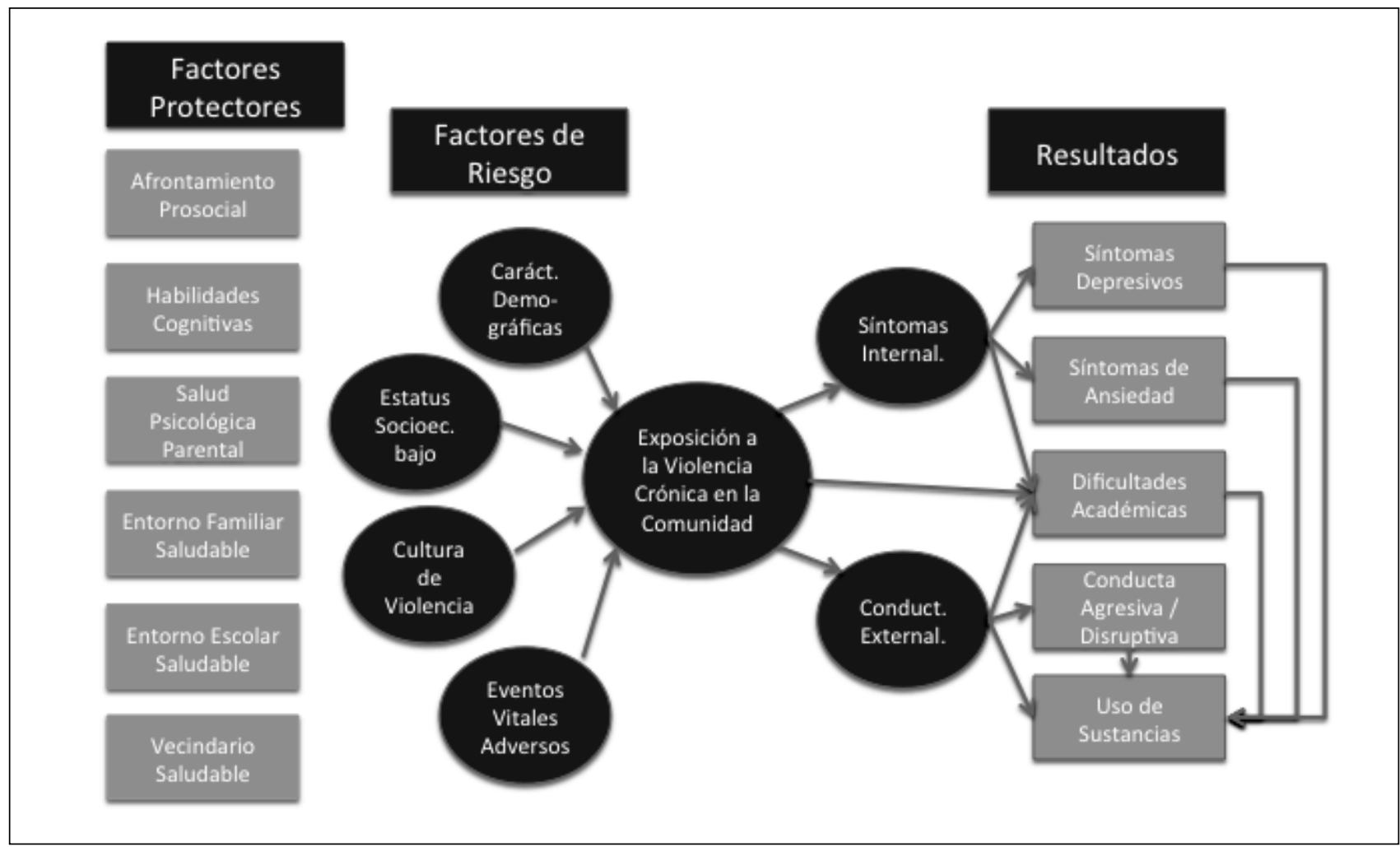


con jóvenes de minoría étnica (ver Cooley-Strickland et al., 2009, para una explicación exhaustiva de los instrumentos y sus propiedades psicométricas).

El modelo conceptual de la Figura 1 determina los análisis que serán realizados utilizando los datos MORE. Los análisis primarios consistirán en evaluar la prevalencia de la exposición a violencia en la comunidad, investigar los factores de riesgo y protección para tal exposición, y examinar los resultados de esa exposición. Entre las cuestiones de interés primario se determinará si los índices de exposición a la violencia difieren por estrato de violencia, género o raza, y si los alumnos con diferentes niveles de factores de riesgo y protección, tales como el apoyo familiar, manifiestan diferentes niveles de exposición a la violencia. Los análisis deberán tener en cuenta el agrupamiento de los alumnos por escuelas y estratos de violencia, utilizando métodos para datos agrupados tales como ecuaciones de estimación generalizadas (GEE; Liang y Zeger, 1986) o modelos multinivel (Raudenbush y Bryk, 2002). Los modelos multinivel permitirán una investigación formal de las relaciones entre las variables tanto a nivel individual del alumno como a nivel escolar y de vecindario, analizando, por ejemplo, cómo los factores de protección tanto de la comunidad, como del individuo reducen la exposición a la violencia. También será importante controlar otras caracterísiticas iniciales de los niños y sus vecindarios, tales como la etnia y la densidad poblacional, puesto que los vecindarios se diferencian no sólo por sus niveles de violencia, sino también por otras características. El uso de modelos GEE y multinivel nos permitirá incluir estas características como predictores adicionales de resultados.

Uno de los puntos fuertes del Proyecto MORE es la disponibilidad de datos de muchas medidas procedentes de múltiples informantes. Esto permite un estudio sistemático de la concordancia entre las respuestas de múltiples informantes, proporcionando una visión adicional de lo que esos individuos experimentan y aportando una guía a los investigadores del ámbito de la violencia en la comunidad para futuras recogidas de datos. El estudio del funcionamiento de los instrumentos empleados en el Proyecto MORE también proporciona una información valiosa sobre el uso de esas medidas en un entorno urbano de ingresos bajos y si los baremos estándar son aplicables.

Otra virtud del Proyecto MORE es la disponibilidad de datos longitudinales de alumnos, con datos disponibles de un periodo de hasta tres años por niño. Esto es poco frecuente en los estudios de violencia en la comunidad, particularmente en el caso de los que emplean múltiples estratos de violencia en el vecindario. Los datos longitudinales permitirán el cálculo de correlaciones simples a lo largo del tiempo, para investigar la estabilidad de la exposición a la violencia, así como los factores de riesgo y protección. También permitirá a los investigadores observar cómo la exposición puntual a la violencia está asociada a resultados observa- dos posteriormente, como ocurre en los casos en que la exposición a la violencia en un año se relaciona con problemas de conducta al año siguiente. La herramienta estadística principal para llevar a cabo estos análisis longitudinales serán de nuevo los modelos GEE y multinivel, que pueden explicar tanto el agrupamiento de observaciones en los niños, como controlar el primer año las características y estadísticas de población (e.g., raza, género) al incluirlas como predictores en los modelos. Modelos GEE más complejos, incluyendo interacciones, permitirán contrastar algunas hipótesis, tales como si las probabilidades de exposición a violencia en la comunidad varían de un panel a otro, si la magnitud de las relaciones varían a lo largo del tiempo, y si las características de base, como la raza/etnia o género, moderan los efectos de exposición a la violencia. En algunos casos, los datos longitudinales podrían ser empleados para analizar las variables mediadoras; por ejemplo, para averiguar si el estrés parental media la relación entre la exposición a la violencia y la conducta de los niños, pero estos análisis deben ser realizados con cautela y deben basarse en gran medida en el modelo conceptual, ya que podría resultar difícil determinar la temporalidad de forma precisa.

\section{La violencia en la comunidad y los jóvenes: tratamiento y prevención}

La necesidad de tratamiento y prevención psicosocial es particularmente acuciante en los barrios pobres, donde los recursos de salud mental son escasos (Cooley y Lambert, 2006). Los efectos de los sucesos traumáticos en los jóvenes disminuyen cuando el joven tiene la oportunidad de procesar esos sucesos (Pynoos y Nader, 1990), y una intervención a edades tempranas es sumamente beneficiosa (Earls, 1991; McAlisterGroves et al., 1993). Por ejemplo, ha habido cierta discusión acerca de si la ansiedad funciona como inhibidor o protector al mantener a los jóvenes que viven en comunidades violentas alejados de la exposición a la violencia (e.g., el miedo podría motivarlos a permanecer en casa). Aunque la evitación en ocasiones es la estrategia más apropiada, los jóvenes necesitan una variedad de estrategias para gestionar las situaciones estresantes y que provocan ansiedad. Aquellos jóvenes con problemas de ansiedad, afectivos y conductuales normalmente poseen un repertorio limitado.

Hay una escasez de estudios de tratamientos de intervención empíricamente validados y publicados sobre el estrés asociado a la violencia en la comunidad. Los padres o cuidadores son importantes mediadores potenciales para prevenir los efectos emocionales adversos de la exposición a violencia en la comunidad (e.g., su disponibilidad física, la aportación de apoyo emocional para superar los sucesos traumáticos, sirviendo como modelos de conducta prosocial y afrontamiento; Wallen y Rubin, 1997). Sin embargo, 
eso presupone que los cuidadores sean modelos de conducta prosocial. El Proyecto MORE investigará si ciertas características de los padres/cuidadores (e.g., hostilidad, ira, trastornos psiquiátricos) ponen en riesgo su habilidad para servir como modelos prosociales. Aunque los padres en el Proyecto MORE informan de la exposición de sus hijos a la violencia en la comunidad, no se incluyen evaluaciones directas de la exposición de los propios padres a dicha violencia, lo cual podría influir en su habilidad para ayudar de forma óptima a sus hijos a enfrentarse a contextos hostiles. El aumento de la competencia de los niños, la autoeficacia, las técnicas de resolución de problemas y la inclusión de los padres en el tratamiento han sido identificados como estrategias potenciales para tratar a los jóvenes que han estado expuestos a la violencia (Davies y Flannery, 1998; Duncan, 1996; Garbarino, Kostelny y Dubrow, 1991). Promover entre los padres un mayor conocimiento del desarrollo infantil (Beardslee et al., 2003) también podría compensar las restricciones que los cuidadores adultos imponen cuando se educa a los niños en entornos con violencia frecuente (Podorefsky, Beardslee y McDonaldDowdell, 2001). Son cruciales los enfoques proactivos porque muchos jóvenes de los barrios pobres no pueden escapar a la exposición directa e indirecta a la violencia en su comunidad (e.g., Garbarino et al., 1991; Osofsky et al., 1993).

Los niños pobres de ciudad son los más vulnerables al desarrollo de problemas emocionales o conductuales (McKay et al., 1998; Tolan y Henry, 1996; Tuma, 1989), pero los que menos probabilidad tienen de recibir servicios adecuados (Day y Roberts, 1991; McKay et al., 1998) y mayor probabilidad de abandonar el tratamiento prematuramente (McKay, McCadam y Gonzales, 1996). Las intervenciones preventivas basadas en la escuela dirigidas a niños expuestos a violencia en la comunidad, particularmente aquéllas que se realizan regularmente fuera de horario escolar y que ofrecen tratamientos intensivos, asequibles, y evolutivamente apropiados, divertidos y basados en evidencia científica son bastante efectivas porque no están estigmatizadas como los programas de educación especial, mantienen a los niños seguros y supervisados en las horas que siguen a las clases y antes de que los padres o cuidadores regresen al hogar tras la jornada laboral, y proporcionan la intensidad necesaria para aplicar adecuadamente la intervención. Este tipo de intervenciones tienen escaso desarrollo en la literatura.

Se han desarrollado una serie de intervenciones con el objetivo de modificar estilos de cognición social, y beneficiar así a los niños expuestos a la violencia en la comunidad. Un programa de este tipo llamado Brain Power (Hudley et al., 1998) fue diseñado para alterar el sesgo atribucional hostil a través de ensayos conductuales. La intervención estaba dirigida a alumnos de tercer a sexto grado y tuvo un impacto positivo en los niños en cuanto a su interpretación de los iguales en situaciones ambiguas. Aunque los sesgos atribucionales eran susceptibles de ser corregidos mediante la intervención, el impacto positivo en la conducta agresiva resultó relativamente efímero. Una intervención más intensiva en grupos pequeños llamada Coping Power ha probado ser capaz de alterar los patrones de procesamiento de información social y conducta agresiva en niños de entre ocho y trece años (Lochman y Wells, 2004). Se observaron efectos similares en la intervención preventiva centrada en aulas escolares y denominada Making Choices, la cual incide en las habilidades de cognición social y el control de emociones de alumnos de escuela elemental (Fraser et al., 2005). Además, el Metropolitan Area Child Study (MACS) evaluó el impacto de la intervención ecológica socio-cognitiva en jóvenes agresivos de comunidades urbanas y barrios pobres (Eron et al., 2002). Este estudio subrayaba la importancia de abordar las actitudes de los jóvenes, sus iguales y padres hacia la violencia para promover cambios duraderos en las creencias de los niños sobre la conveniencia y efectividad de la agresión.

Los programas de prevención e intervención poseen habitualmente un impacto mínimo en la producción de disuasores de violencia juvenil duraderos (Tolan y Guerra, 1994) y en sus efectos, aunque quizás logren generar mejores expectativas para las víctimas de violencia en las comunidades. Es esencial, sin embargo, identificar en su totalidad las múltiples influencias que afectan las vidas de los niños urbanos (e.g., Reese, Vera, Thompson y Reyes, 2001). Por ejemplo, resulta problemático enseñar habilidades de manejo de conflicto a los jóvenes urbanos sin abordar los efectos de ser testigos de violencia (Jenkins y Bell, 1994). La teoría ecológica del desarrollo humano enfatiza la importancia de entender a los niños en su contexto, elemento esencial a la hora de diseñar intervenciones efectivas de tratamiento y prevención (e.g., Bronfenbrenner, 1979; Lerner, 1995; Lewis, Lewis, Daniels y D’Andrea, 1998). Así mismo, atender a las diferencias culturales cuando se interviene con los jóvenes es esencial para mejorar la intervención (Kazdin, 1993). Cada comunidad posee unas características, unos puntos fuertes y necesidades culturales únicas; es importante "customizar" las intervenciones cuando se trabaja con poblaciones específicas (Reese et al., 2001). Se precisan estudios bien diseñados, para desarrollar intervenciones preventivas y de tratamiento efectivas en el ámbito escolar.

\section{Agradecimientos}

Los autores quieren mostrar su agradecimiento a todos aquéllos que han colaborado en la investigación del Proyecto MORE. También agradecemos el trabajo de nuestros colaboradores: Scott Hubbard, Nicholas Ialongo, Phillip Leaf, Megan Bair-Merrit, y Jean Ko. 
Esta tarea ha sido sólo posible gracias al continuo apoyo y cooperación de la Red de Escuelas Públicas de la Ciudad de Baltimore y a nuestros seis centros escolares colaboradores. Los administradores y la plantilla de estas escuelas han proporcionado acceso y orientación, permitiéndonos aprender de ellos. Queremos agradecer sinceramente a los estudiantes de la Ciudad de Baltimore y a sus familias, que han compartido sus vidas con nosotros para mejorar la situación de otros. Agradecemos además los esfuerzos del entregado equipo MORE (Alisa, Amber, Andrew, Corina, Dan, Debbie, Katie, Maria, Max, Mike, Lindsay, Steph, Ty y Winn), con mención especial de Kathryn Otte por sus contribuciones a este estudio. El apoyo económico para el Proyecto MORE proviene de una beca otorgada por el National Institute on Drug Abuse a M. Cooley (1 R01 DA018318).

\section{Referencias}

Aisenberg, E. y Ell, K. (2005). Contextualizing community violence and its effects: An ecological model of parentchild interdependent coping. Journal of Interpersonal Violence, 20, 855-871.

Alexander, K. L., Entwisle, D. R. y Kabbani, N. S. (2001). The dropout process in life course perspective: Early risk factors at home and school. Teachers College Record, 103, 760-822.

Attar, B. K. y Guerra, N. G. (1994). The effects of cumulative violence exposure on children living in urban neighborhoods. Paper presented at the meeting of the American Psychological Society Convention, Washington, D.C.

Attar, B. K., Guerra, N. C. y Tolan, P. H. (1994). Neighborhood disadvantage, stressful life events, and adjustment in urban elementary school children. Journal of Clinical Child Psychology, 23, 391-400.

Bandura, A. (1973). Aggression: A social learning analysis. Englewood Cliffs, NJ: Prentice Hall.

Bandura, A., Ross, D. y Ross, S. (1961). Transmission of aggression through imitation of aggressive models. Journal of Abnormal and Social Psychology, 63, 575582.

Barreto, S. y McManus, M. (1997). Casting the net for "depression" among ethnic minority children from highrisk urban communities. Clinical Psychology Review, 17, 823-845.

Beardslee, W. and Associates (2003). Hope, Meaning and Continuity: A Program for Helping Families when Parents Face Depression. Boston, MA: Judge Baker's Children's Center.

Beardslee, W. y Podorefsky, D. (1988). Resilient adolescents whose parents have serious affective and other psychiatric disorders: The importance of self-understanding and relationships. American Journal of Psychiatry, 145, 6369.

Beardslee, W., Versage, E. y Gladstone, T. (1998). Children of affectively ill parents: A review of the past ten years.
Journal of the American Academy of Child and Adolescent Psychiatry, 37, 1134-1141.

Bell, C. (1997). Community violence: Causes, prevention, and intervention. Journal of the National Medical Association, 89, 657-662.

Bell, C. C. y Jenkins, E. J. (1991). Traumatic stress and children. Journal of Health Care for the Poor and Undeserved, 2, 175-188.

Bolton, D., O'Ryan, D., Udwin, O., Boyle, S. y Yule, W. (2000). The long-term psychological effects of a disaster experienced in adolescence: II: General psychopathology. Journal of Child Psychology and Psychiatry, 41, 513523.

Boney-McCoy, S. y Finkelhor, D. (1995). Prior victimization: A risk factor for child sexual abuse and for PTSDrelated symptomatology among sexually abused youth. Child Abuse and Neglect, 19, 1401-1421.

Bowen, N. K. y Bowen, G. L. (1999). Effects of crime and violence in neighborhoods and schools on the school behavior and performance of adolescents. Journal of Adolescent Research, 14, 319-342.

Boyd, R., Cooley, M., Lambert, S. y Ialongo, N. (2003). First grade child risk behaviors for community violence in middle school. Journal of Community Psychology, 31, 297-314.

Bradshaw, C. P. y Garbarino, J. (2004). Social cognition as a mediator of the influence of family and community violence on adolescent development: Implications for intervention. En J. Devine, J. Gilligan, K. A. Miczek, R. Shaikh, D. Pfaff (Eds.), Youth violence: Scientific approaches to prevention (pp. 85-105). New York: New York Academy of Sciences.

Bronfenbrenner, U. (1979). The Ecology of Human Development. Cambridge, MA: Harvard University Press.

Brooks, P. H. y Kendall, E. D. (1982). Working with children. En R. Vasta (Ed.), Strategies and Techniques of Child Study (pp. 325-343). New York: Academy.

Brown, D., Ahmed, F., Gary, L. y Milburn, N. (1995). Major depression in a community sample of African Americans. American Journal of Psychiatry, 152, 373-378.

Bruce, M., Takeuchi, D. y Leaf, P. (1991). Poverty and psychiatric status: Longitudinal evidence from the New Haven Epidemiologic Catchment Area Study. Archives of General Psychiatry, 48, 470-474.

Brunswick, A. y Messeri, P. (1984). Origins of cigarette smoking in academic achievement, stress and social expectations: Does gender make a difference? Journal of Early Adolescence, 4, 353-370.

Bryant, A., Schulenberg, J., Bachman, J., O'Malley, P. y Johnston, L. (2000). Understanding the links among school misbehavior, academic achievement, and cigarette use: A national panel study of adolescents. Prevention Science, 1, 71-87.

Bureau of Justice Statistics. (1991). Criminal victimization in the United States, 1989. Washington, DC: US Department of Justice.

Burns, M., Morrison, J., Khoury, P. y Glueck, C. (1980). 
Blood pressure studies in Black and White inner-city and suburban adolescents. Preventive Medicine, 9, 41-50.

Christofel, K. (1990). Violent death and injury in US children and adolescents. American Journal of Disease Control, 144, 697-706.

Cooley-Quille, M. R. y Lorion, R. (1999). Adolescents' exposure to community violence: Sleep and psychophysiological functioning. Journal of Community Psychology, 27, 367-375.

Cooley-Quille, M. R., Turner, S. M. y Beidel, D. C. (1995). The emotional impact of children's exposure to community violence: A preliminary study. Journal of the American Academy of Child and Adolescent Psychiatry, 34, 1362-1368.

Cooley-Quille, M., Boyd, R. C., Frantz, E. y Walsch, J. (2001). Emotional and psychophysiological impact of exposure to community violence in urban adolescents. Journal of Child Clinical Psychology, Special Section: Violence y Youth, 30, 199-206.

Cooley-Strickland, M. Quille, T. J., Griffin, R. S., Stuart, E. A., Bradshaw, C. P. y Furr-Holden, D. (2009). Community violence and youth: Affect, behavior, substance use, and academics. Clinical Child and Family Psychology Review, 12, 127-156.

Cooley, M. y Boyd, R. (2009). Assessing youth and parents' exposure to community violence: Extensions of the CREV. Manuscript in preparation.

Cooley, M. R., Turner, S. M. y Beidel, D. C. (1995). Assessing community violence: The Children's Report of Exposure to Violence (CREV). Journal of the American Academy of Child and Adolescent Psychiatry, 34, 201208.

Cooley, M. y Lambert, S. (2006). School-based interventions for children and adolescents exposed to chronic community violence. En A. Lieberman, R. DeMartino y N. Fox (Eds.), Interventions for Children Exposed to Violence. New Jersey: Johnson y Johnson Pediatric Institute.

Cooley, M., Boyd, R. C. y Grados, J. J. (2004). Feasibility of an anxiety preventive intervention for community violence exposed children. Journal of Primary Prevention, 25, 105-123.

Crick, N. R. y Dodge, K. A. (1994). A review and reformulation of social information processing mechanisms in children's social adjustment. Psychological Bulletin, 115, 74-101.

Crouch, J. L., Hanson, R. F., Saunders, B. E., Kilpatrick, D. G. y Resnick, H. S. (2000). Income, race/ethnicity, and exposure to violence in youth: Results from the national survey of adolescents. Journal of Community Psychology, 28, 625-641.

Davies, W. H. y Flannery, D. J. (1998). Post-traumatic stress disorder in children and adolescents exposed to violence. Pediatric Clinics of North America, 45, 341-353.

Day, C. y Roberts, M. (1991). Activities of the Child and Adolescent Service System Program for improving mental health services for children and families. Journal of Clinical Child Psychology, 20, 340-350.
Dodge, K. A. y Pettit, G. S. (2003). A biopsychosocial model of the development of chronic conduct problems in adolescence. Developmental Psychology, 39, 349-371.

Dodge, K. A. y Somberg, D. R. (1987). Hostile attributional biases among aggressive boys are exacerbated under conditions of threat. Child Development, 58, 213-224.

Dodge, K. y Frame, C. L. (1982). Social-cognitive biases and deficits in aggressive boys. Child Development, 53, 620-635.

Dossey L. (1993). Healing Words. San Francisco: Harper.

Duncan, D. F. (1996). Growing up under the gun: Children and adolescents coping with violent neighborhoods. The Journal of Primary Prevention, 16, 343-356.

Duncan, S., Duncan, T. y Strycker, L. (2000). Risk and protective factors influencing adolescent problem behavior: A multivariate latent growth curve analysis. Annals of Behavioral Medicine, 22, 103-109.

DuRant, R., Cadenhead, C., Pendergrast, R., Slavens, G. y Linder, C. (1994). Factors associated with the use of violence among Black adolescents. American Journal of Public Health, 84, 612-617.

Earls, F. (1991). A developmental approach to understanding and controlling violence. En H. Fitzgerald et al. (Eds.), Theory and Research in Behavioral Pediatrics (pp. 6188). New York: Plenum.

Ellickson, P. y Morton, S. (1999). Identifying adolescents at risk for hard drug use: Racial/ethnic variations. Journal of Adolescent Health, 25, 382-395.

Ellison, C. G. (1991). Religious involvement and subjective well-being. Journal of Health and Social Behavior, 32, 80-99.

Epstein, J., Botvin, G., Diaz, T., Williams, C. y Griffin, K. (2000). Aggression, victimization and problem behavior among inner city minority adolescents. Journal of Child and Adolescent Substance Abuse, 9, 51-66.

Eron, L., Huesmann, R., Spindler, A., Guerra, N., Henry, D. y Tolan, P. (2002). A cognitive-ecological approach to preventing aggression in urban settings: Initial outcomes for high-risk children. Journal of Consulting and Clinical Psychology, 70, 179-194.

Everett, S., Giovino, G., Warren, C., Crossett, L. y Kann, L. (1998). Other substance use among high school students who use tobacco. Journal of Adolescent Health, 23, 289296.

Farrell, A. D. y Bruce, S. E. (1997). Impact of exposure to community violence on violent behavior and emotional distress among urban adolescents. Journal of Clinical Child Psychology, 26, 2-14.

Fitzpatrick, K. (1993). Exposure to violence and presence of depression among low-income, African-American youth. Journal of Consulting and Clinical Psychology, 61, 528531.

Fitzpatrick, K. M. y Boldizar, J. P. (1993). The prevalence of depression among low-income African American youth. Journal of Consulting and Clinical Psychology, 61, 528531.

Fraser, M. W., Galinsky, M. J., Smokowski, P. R., Day, S. H., Terizan, M. A., Rose, R. A. y Guo, S. (2005). Social infor- 
mation-processing skills training to promote social competence and prevent aggressive behaviors in the third grade. Journal of Consulting and Clinical Psychology, 73, 1045-1055.

Garmezy, N. (1993). Children in poverty: Resilience despite risk. Psychiatry: Interpersonal and Biological Processes, 56, 127-136.

Garbarino, J., Kostelny, K. y Dubrow, N. (1991). No place to be a child: Growing up in a war zone. San Francisco: Jossey-Bass.

Giaconia, R. y Reinherz, H. (1995). Traumas and posttraumatic stress disorder in a community population of older adolescents. Journal of the American Academy of Child $y$ Adolescent Psychiatry, 34, 1369.

Gladstein, J., Rusonis, E. J. y Heald, F. P. (1992). A comparison of inner-city and upper-middle class youths' exposure to violence. Journal of Adolescent Health, 13, 275280.

Gorman-Smith, D. y Tolan, P. (1998). The role of exposure to community violence and developmental problems among inner-city youth. Development and Psychopathology, 10, 101-116.

Guerra, N. G., Huesmann, L. R. y Spindler, A. (2003). Community violence exposure, social cognition, and aggression among urban elementary school children. Child Development, 74, 1561-1576.

Hampton, R., Gelles, R. y Harrop, J. (1989). Is violence in black families increasing? A comparison of 1975 and 1985 national survey rates. Journal of Marriage and the Family, 51, 969-980.

Hinton-Nelson, M. D., Roberts, M. C. y Snyder, C. R. (1996). Early adolescents exposed to violence: Hope and vulnerability to victimization. American Journal of Orthopsychiatry, 66, 346-353.

Hollman, C. M. y McNamara, J. R. (1999). Considerations in the use of active and passive parental consent procedures. The Journal of Psychology, 133, 141-156.

Horn, J. L. y Trickett, P. K. (1998). Community violence and child development: A review of research. En P. Trickett y C. Schellenbach (Eds.), Violence Against Children in the Family and the Community (pp. 103-138). Washington, DC: American Psychological Association.

Hudley, C., Britsch, B., Wakefield, W. D., Smith, T., Demorat, M. y Cho, S. J. (1998). An attribution retraining program to reduce aggression in elementary school students. Psychology in the Schools, 35, 271-282.

Huizinga, D., Loeber, R. y Thornberry, T. (1993). Longitudinal study of delinquency, drug use, sexual activity, and pregnancy among children and youth in three cities. Public Health Reports: Journal of the U.S. Public Health Service, 108, 90-96.

Ialongo, N., Edelsohn, G., Werthamer-Larsson, L., Crockett, L. y Kellam, S. (1996). The course of aggression in firstgrade children with and without co-morbid anxious symptoms. Journal of Abnormal Child Psychology, 24, 445-456.

Institute of medicine. (1994). New directions in definitions. En P. J. Mrazek y R. J. Haggerty (Eds.), Reducing Risks for Mental Disorders: Frontiers for Preventive Intervention Research (pp. 19-29). Washington, DC: National Academy Press.

Jenkins, E. J. (2001). Violence and trauma in the lives of African American children. En A. Neal-Barnett, J. Contreras y K. Kerns (Eds.), Forging Links: African American Children Clinical Developmental Perspectives (pp. 107-128). Westport, CT: Praeger.

Jenkins, E. J. y Bell, C. G. (1994). Post-traumatic stress disorder and violence among inner city high school students. En S. Friedman (Ed.), Anxiety disorders in African Americans. New York: Springer.

Jessor, R., van den Boss, J., Vanderryn, J., Costa, F. y Turbin, M. (1995). Protective factors in adolescent problem behavior: Moderator effects and developmental change. Developmental Psychology, 31, 923-933.

Jones, J. y Barlow, D. H. (1990). The etiology of post-traumatic stress disorder. Clinical Psychology Review, 10, 299-328.

Kandel, D., Kessler, R. y Margulies, R. (1978). Antecedents of adolescent initiation into stages of drug use: A developmental analysis. Journal of Youth and Adolescence, 7, 13-40.

Kashani, J. H., Deuser, W. y Reid, J. C. (1991). Aggression and anxiety: A new look at an old notion. Journal of the American Academy of Child and Adolescent Psychiatry, 30, 218-223.

Kazdin, A. E. (1993). Adolescent mental health. American Psychologist, 48, 127-141.

Keane, T. M., Gerardi, R. J., Lyons, J. A. y Wolfe, J. (1988). The interrelationship of substance abuse and PTSD: Epidemiological and clinical considerations. En M. Galanter (Ed.), Recent developments in alcoholism, Vol. V. New York: Plenum Press.

Kendall, P. C. y Hammen, C. (1995). Abnormal Psychology. Boston, MA: Houghton Mifflin Co.

Kilpatrick, D. G., Acierno, R., Saunders, B., Resnick, H. S., Best, C. L. y Schnurr, P. P. (2000). Risk factors for adolescent substance abuse and dependence: Data from a national sample. Journal of Consulting and Clinical Psychology, 68, 19-30.

Kliewer, W., Lepore, S. J., Oskin, D. y Johnson, P. D. (1998). The role of social and cognitive processes in children's adjustment to community violence. Journal of Consulting and Clinical Psychology, 66, 199-209.

Koenig, H. G. (1997). Use of religion by patients with severe medical illness. Mind/Body Medicine, 2, 31-36.

Ladd, G. W. y Burgess, K. B. (1999). Charting the relationship trajectories of aggressive, withdrawn, and aggressive/withdrawn children during early grade school. Child Development, 70, 910-929.

Ladd, G. W. y Kochenderfer-Ladd, B. J. (2002). Identifying victims of peer aggression: Analysis of cross-informant data for concordance, estimation of relational adjustment, prevalence of victimization. Psychological Assessment, $14,1-23$.

LaGreca, A., Silverman, W. y Wasserstein, S. (1998). Children's pre-disaster functioning as a predictor of post- 
traumatic stress following hurricane Andrew: A prospective study. Journal of Consulting and Clinical Psychology, 64, 712-723.

Leonard, H. L., Goldberger, E. L., Rapoport, J. L., Cheslow, D. L. y Swedo, S. E. (1990). Childhood rituals: Normal development or obsessive-compulsive symptoms? Journal of the American Academy of Child and Adolescent Psychiatry, 29, 17-23.

Lerner, R. (1995). America's youth in crisis: Challenges and options for programs and policies. Thousand Oaks, CA: Sage.

Lewis, J. A., Lewis, M. D., Daniels, J. H. y D’Andrea, M. J. (1998). Community counseling: Empowerment strategies for a diverse society. Pacific Grove, CA: Brooks-Cole.

Liang, K-Y. y Zeger, S. L. (1986). Longitudinal data analysis using generalized linear models. Biometrika, 73, 13-22.

Linares, L.O., Heeren, T., Bronfman, E., Zuckerman, B., Augustyn, M. y Tronick, E. (2001). A meditational model for the impact of exposure to community violence on early child behavior problems. Child Development, 72, 639-652.

Lipsitz, S. R., Molenberghs, G., Fitzmaurice, G. M. y Ibrahim, J. (2000). GEE with Gaussian estimation of the correlations when data are incomplete. Biometrics, 56, 528-536.

Lochman, J. E. y Wells, K. C. (2004). The Coping Power program for preadolescent aggressive boys and their parents: Outcome effects at the 1-year follow-up. Journal of Consulting and Clinical Psychology, 4, 571-578.

Loeber, R. y Keenan, K. (1994). Interaction between conduct disorder and its comorbid conditions: Effects of age and gender. Clinical Psychology Review, 14, 497-523.

Lorion, R. (1998). Exposure to pervasive community violence: Contamination of the school environment. En D. S. Elliott, K. Williams y B. Hamburg (Eds.), Violence in American Schools (pp. 293-311). New York: Cambridge University Press.

Lorion, R. L., Brodsky, A. y Cooley-Quille, M. R. (1999). Exposure to urban violence: A framework for conceptualizing risky settings. En D. E. Biegel y A. Blum (Eds.), Innovations in Practice and Service Delivery across the Lifespan (pp. 124-143). New York: Oxford.

Luthar, S., Cicchetti, D. y Becker, B. (2000). The construct of resilience: A critical evaluation and guidelines for future work. Child Development, 71, 543-562.

Mackesy-Amiti, M. y Fendrich, M. (2000). Trends in inhalant use among high school students in Illinois: 19931995. American Journal of Drug and Alcohol Abuse, 26, 569-590.

March, J. S. y Parker, J. D. (1999). The Multidimensional Anxiety Scale for Children (MASC). En M. Maruish (Ed.), The Use of Psychological Testing or Treatment Planning and Outcomes Assessment (2 $2^{\text {nd }}$ ed.) pp. 299322. Mahwah, NJ: Lawrence Erlbaum.

Marks, I. (1987). Fears, phobias and rituals. NY: Oxford University Press.

Martinez, P. y Richters, J. E. (1993). The NIMH Community
Violence Project: II. Children's distress symptoms associated with violence exposure. Psychiatry: Interpersonal and Biological Processes, 56, 22-35.

McAlister-Groves, B., Zuckerman, B., Marans, B. y Cohen, D. (1993). Silent victims: Children who witness violence. Journal of the American Medical Association, 269, 262264.

McKay, M., McCadam, K. y Gonzales, J. (1996). Addressing the barriers to mental health services for inner-city children and their caretakers. Community Mental Health Journal, 32, 353-361.

McKay, M., Stoewe, J., McCadam, K. y Gonzales, J. (1998). Increasing access to mental health services for urban children and their caregivers. Health and Social Work, 23, 915.

Mowrer, O. H. (1960). Learning theory and the symbolic processes. New York: Wiley.

Mulvey, E., Odgers, C., Skeem, J., Gardner, W., Schubert, C. y Lidz, C. (2006). Substance Use and Community Violence: A Test of the Relation at the Daily Level. Journal of Consulting and Clinical Psychology, 74, 743754.

Mushe-Eizenman, D. R., Boxer, P., Danner, S., Dubow, E. F., Goldstein, S. E. y Heretick, D. M. L. (2004). Social-cognitive mediators of the relation of environmental and emotion regulation factors to children's aggression. Aggressive Behavior, 30, 389-408.

Obot, I. y Anthony, J. (2000). School dropout and injecting drug use in a national sample of White non-Hispanic American adults. Journal of Drug Education, 30, 145155.

Osofsky, J. D. (1995). The effects of exposure to violence on young children. American Psychologist, 50, 782-788.

Osofsky, J. D., Werers, S., Hann, D. M. y Fick, A. C. (1993). Chronic community violence: What is happening to our children? Psychiatry, 56, 36-45.

Overstreet, S. y Braun, S. (1999). A preliminary examination of the relationship between exposure to community violence and academic functioning. School Psychology Quarterly, 14, 380-396.

Parker, R. y Auerhahn, K. (1998). Alcohol, drugs, and violence. Annual Review of Sociology, 24, 291-311.

Podorefsky, D., Beardslee, W. y McDonald-Dowdell, M. (2001). Adaptation of preventive interventions for use in a low-income culturally diverse community. Journal of the American Academy of Child and Adolescent Psychiatry, 40, 879-886.

Pynoos, R. S. y Nader, K. (1990). Children's exposure to violence and traumatic death. Psychiatry Annals, 20, 334344.

Pynoos, R. S., Frederick, C., Nader, K. y Arroyo, W. (1987). Life threat and post-traumatic stress in school-age children. Archives of General Psychiatry, 44, 1057-1063.

Pynoos, R., Steinberg, A. y Piacentini, J. (1999). A developmental psychopathology model of childhood traumatic stress and intersection with anxiety disorders. Biological Psychiatry, 46, 1542-1554.

Raudenbush, S. W. y Bryk, A. S. (2002). Hierarchical lin- 
ear models: Applications and data analysis methods, $2^{\text {nd }}$ Edition. Thousand Oaks, CA: Sage.

Reese, L. E., Vera, E. M., Thompson, K. y Reyes, R. (2001). A qualitative investigation of perceptions of violence risk factors in low-income African American children. Journal of Clinical Child Psychology, 30, 199-206.

Resnick, M., Bearman, P., Blum, R., Bauman, K., Harris, K., Jones, J. ... Udry, J. R. (1997). Protecting adolescents from harm: Findings from the National Longitudinal Study on Adolescent Health. Journal of the American Medical Association, 278, 823-832.

Richters, J. E. y Martinez, P. (1993). The NIMH community violence project:. Children as victims and witnesses to violence. Psychiatry, 56, 7-21.

Rutter, M. (1987). Psychosocial resilience and protective mechanisms. American Journal of Orthopsychiatry, 57, 316-331.

Rutter, M. y Quinton, D. (1977). Psychiatric disorder: Ecological factors and concepts of causation. En $\mathrm{H}$. McGurk (Ed.), Ecological factors in human development (pp. 173-187). Amsterdam: North-Holland.

Saltzman, W. R. (1996). Exposure to community violence and the prediction of violent antisocial behavior in a multi-ethnic sample of adolescents (Doctoral dissertation, University of Maryland, College Park, 1996). Dissertation Abstracts International: Section: The Sciences and Engineering, 57, 2223.

Sameroff, A., Bartko, W., Baldwin, A., Baldwin, C. y Seifer, R. (1998). Introduction: Family and social influences on the development of child competence. En M. Lewis y C. Feiring (Eds.), Families, Risk, and Competence (pp. 161185). Mahwah, NJ: Erlbaum.

Scheeringa, M. y Zeanah, C. (1995). Symptom expression and trauma variables in children under 48 months of age. Infant Mental Health Journal, 16, 259-270.

Schubiner, H., Scott, R. y Tzelepis, A. (1993). Exposure to violence among inner-city youth. Journal of Adolescent Health, 14, 214-219.

Schwab-Stone, M. E., Ayers, T. S., Kasprow, W., Voyce, C., Barone, C., Shriver, T. y Weissberg. R. P. (1995). No safe haven: A study of violence exposure in an urban community. Journal of the American Academy of Child and Adolescent Psychiatry, 34, 1343-1352.

Schwab-Stone, M., Chen, C., Greenberger, E., Silver, D., Lichtman, J. y Voyce, C. (1999). No safe haven II: The effects of violence exposure on urban youth. Journal of the American Academy of Child y Adolescent Psychiatry, 38, 359-367.

Schwartz, D. y Gorman, A. (2003). Community violence exposure and children's academic functioning. Journal of Educational Psychology, 95, 163-173.

Schwarz, E. D. y Kowalski, J. M. (1991). Malignant memories: PTSD in children and adults after a school shooting. Journal of the American Academy of Child and Adolescent Psychiatry, 30, 936-944.

Schwarz, N. (1999). Self-reports: How the questions shape the answers. American Psychologist, 54, 93-105.

Selner-O’Hagan, M. B., Kindlon, D. J., Buka, S. L.,
Raudenbush, S. W. y Earls, F. J. (1998). Assessing exposure to violence in urban youth. Journal of Child Psychology and Psychiatry and Allied Disciplines, 39, 215-224.

Shahinfar, A., Fox, N. y Leavitt, L. (2000). Preschool children's exposure to violence: Relation of behavior problems to parent and child reports. American Journal of Orthopsychiatry, 70, 115-125.

Shakoor, B. H. y Chalmers, D. (1991). Co-victimization of African-American children who witness violence: Effects on cognitive, emotional, and behavioral development. Journal of the National Medical Association, 83, 233238.

Swanson, C. B. (2004). Projections of 2003-04 High School Graduates: Supplemental analyses based on findings from Who Graduates? Who Doesn't? Retrieved April 20, 2009, http://www.urban.org/publications/411019.html

Taylor, R. J., Tucker M. B., Chatters L. M. y Jayakody R. (1997). Recent demographic trends in African American family structure. En R. Taylor, J. Jackson y L. M. Chatters (Eds.), Family Life in Black America (pp. 14-62). Thousand Oaks, CA: Sage Publications.

Thomas, S. P. y Groer, M.W. (1986). Relationship of demographic, life-style and stress variables to blood pressure in adolescents. Nursing Research, 35, 169-172.

Tiet, Q., Bird, H., Davies, M., Hoven, C., Cohen, P., Jensen, P. y Goodman, S. (1998). Adverse life events and resilience. Journal of the American Academy of Child and Adolescent Psychiatry, 37, 1191-1200.

Tolan, P. H., Guerra, N. G. y Montaini-Klovdahl, L. R. (1997). Staying out of harm's way: Coping and the development of inner-city children. En S. Wolchik y I. Sandler (Eds.), Handbook of Children's Coping: Linking Theory and Intervention. New York: Plenum Press.

Tolan, P. y Guerra, N. (1994). Prevention of delinquency: Current status and issues. Applied y Preventive Psychology, 3, 251-273.

Tolan, P. y Henry, D. (1996). Patterns of psychopathology among urban poor children: Co-morbidity and aggression effects. Journal of Consulting and Clinical Psychology, 64, 1094-1099.

Tuma, J. (1989). Mental health services for children: The state of the art. American Psychologist, 44, 188-198.

Turner, J. y Lloyd, D. (1999). The stress process and the social distribution of depression. Journal of Health and Social Behavior, 40, 374-404.

Turner, S., Beidel, D. y Cooley-Quille, M. (1997). Social Effectiveness Therapy: A Therapist's Guide. Toronto: MHS.

Uehara, E., Chalmers, D., Jenkins, E. J. y Shakoor, B. (1996). African-American youth encounters with violence: Results from the Chicago community mental health council violence project. Journal of Black Studies, 26, 768-781.

Wallen, J. y Rubin, R. H. (1997). The role of the family in mediating the effects of community violence on children. Aggression and Violent Behavior, 2, 33-41.

Walters, S. T. y Bennett, M. E. (2000). Assessing client's 
spirituality and religious behavior: Recommendations for research and practice in mental health settings. The Behavior Therapist, 23, 79-90.

Weisz, J., Weiss, B., Wasserman, A. y Rintoul, B. (1987). Control-related beliefs and depression among clinicreferred children and adolescents. Journal of Abnormal Psychology, 96, 58-63.

White, K. S., Bruce, S. E., Farrell, A. D. y Kliewer, W. (1998). Impact of exposure to community violence on anxiety: A longitudinal study of family social support as a protective factor for urban children. Journal of Child and Family Studies, 7, 187-203.

Wills, T., Sandy, J. y Yaeger, A. (2001). Time perspective and early-onset substance use: A model based on stresscoping theory. Psychology of Addictive Behaviors, 15, 118-125.

World Health Organization (2002). World Report on Violence and Health. Brussels, Belgium: WHO.

Manuscrito Recibido: 18/02/2011

Revisión Recibida: 25/05/2011

Manuscrito Aceptado: 07/06/2011 